\title{
Inclusión de los ODS en la enseñanza-aprendizaje de la Ciencia del Suelo
}

Cristina Lull Noguera ${ }^{a}$, Inmaculada Bautista Carrascosa ${ }^{b}$, Antonio Lidón Cerezuela ${ }^{c}$, Josep Llinares Palacios $^{\mathrm{d}}, \mathbf{M}^{\mathrm{a}}$ Desamparados Soriano Soto ${ }^{\mathrm{e}}$

aDpto. Química, clull@upvnet.upv.es; ${ }^{b}$ Dpto. Química, ibautista@qim.upv.es; 'Dpto. Química, alidon@qim.upv.es; ${ }^{\mathrm{d}}$ Dpto. Química, jollipa@qim.upv.es; 'Dpto. Producción vegetal, asoriano@prv.upv.es. Universitat Politècnica de València.

\section{\$EWWDW}

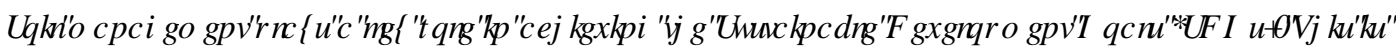

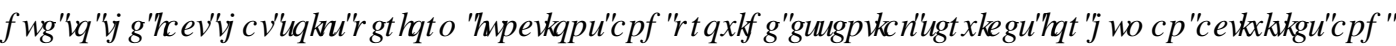

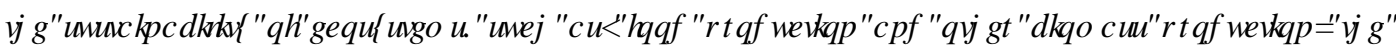

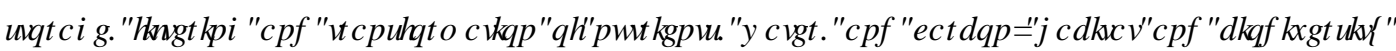

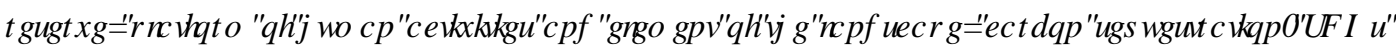

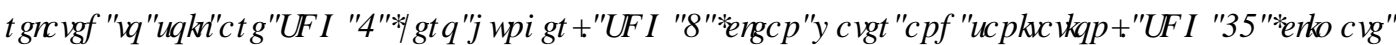

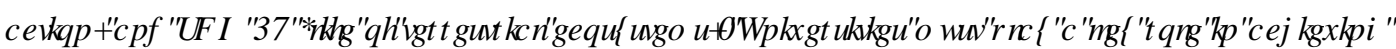

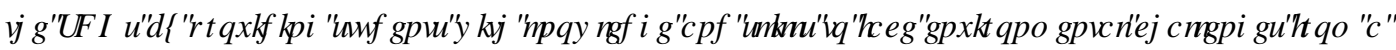

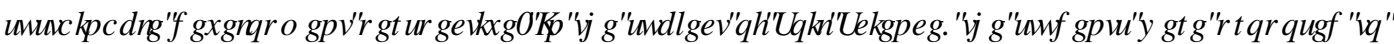

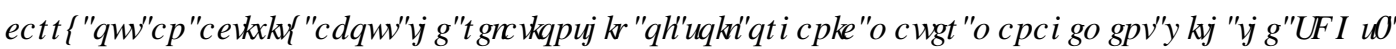

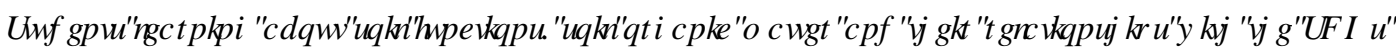

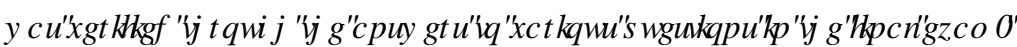

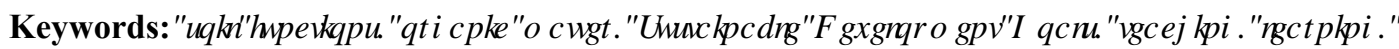
HDOXDURQD

\section{HXP HQ}

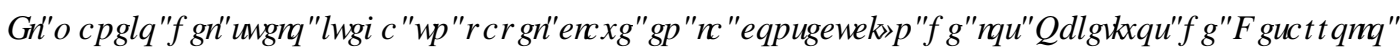

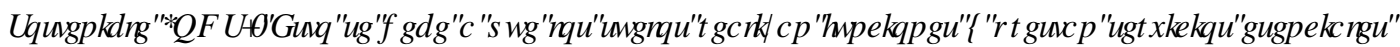

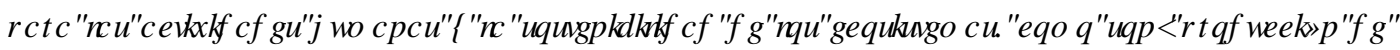

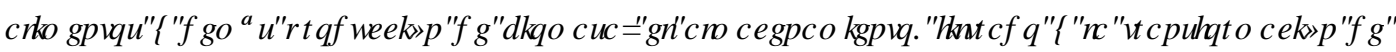

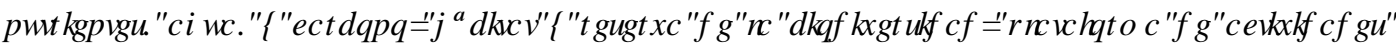

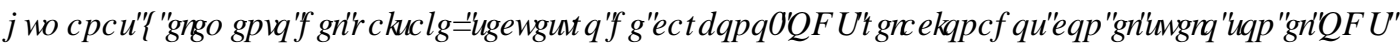

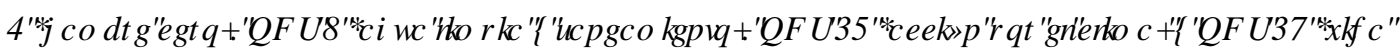

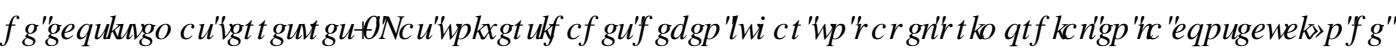

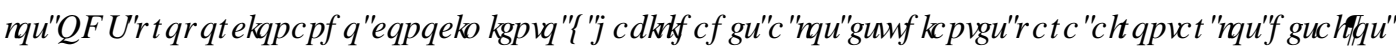

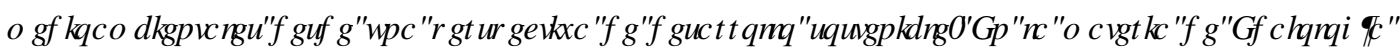

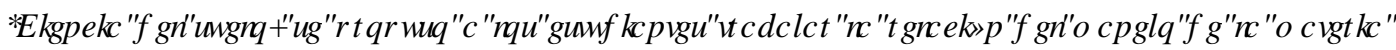

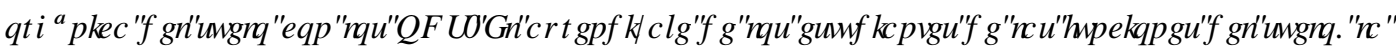

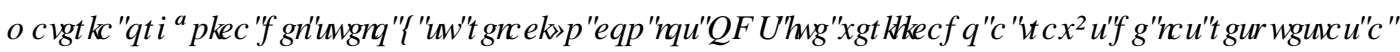
YDUDVSUH XQWVHQHOH DP HQIIQDOD

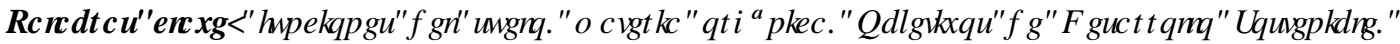
HQM KDQ] DIDSLHQG] DNFIIHMDODFIy QW 


\section{Introducción}

En 2015, en la Cumbre del Desarrollo Sostenible se aprobó la Agenda 2030. Esta Agenda tiene como núcleo central 17 Objetivos de Desarrollo Sostenible (ODS) (Figura 1) de aplicación universal que no son jurídicamente obligatorios. La Agenda 2030 con sus 17 ODS representa el compromiso internacional para hacer frente a los retos sociales, económicos y medioambientales de la globalización, poniendo en el centro a las personas, el planeta, la prosperidad y la paz. La Agenda 2030 es una oportunidad para que los países, instituciones, empresas y ciudadanos, emprendamos un nuevo camino con el que mejorar la vida de todos, sin dejar a nadie atrás. El objetivo de los ODS es transformar el mundo logrando un modo de vida más sostenible. Entre los desafíos de los ODS se encuentran: la eliminación de la pobreza y el hambre, el combate al cambio climático, la garantía de una educación inclusiva, equitativa y de calidad, la defensa de la igualdad de la mujer, la protección del medio ambiente o el diseño de ciudades más inclusivas, seguras, resilientes y sostenibles (Figura 1). Es importante que toda la comunidad universitaria se dé cuenta de la importancia del cumplimiento integral de los ODS y más teniendo en cuenta que son interdependientes entre sí.

\begin{tabular}{|c|c|c|c|c|c|}
\hline $\begin{array}{l}\text { OBJETIVES } \\
\text { DE DESARROLLO } \\
\text { SOSTENIBLE }\end{array}$ & 1 DELLA POBREZA & $2 \stackrel{\text { GerRO }}{\text { HaMBRE }}$ & 3 Salud & $4 \stackrel{\text { DE CALCADAD }}{\text { EDCÁN }}$ & 5 \\
\hline 6 YGSANEAMIIETTO & 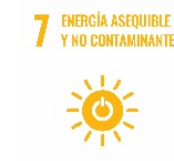 & 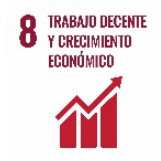 & 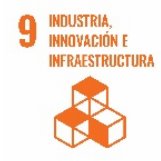 & 10 RESUCUION NEEAS & 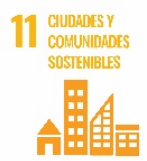 \\
\hline 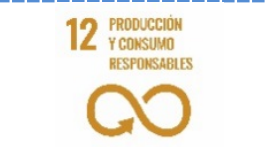 & 13 PORE Cl CluAA & $\underbrace{14}$ & 15 VIDA & 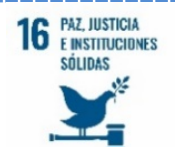 & $\begin{array}{l}17 \text { LlaAvZA Para } \\
\text { LoS OBJEIIvos }\end{array}$ \\
\hline
\end{tabular}

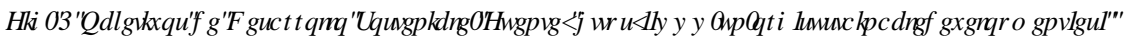

Los 17 ODS se concretan en 169 metas y en 230 indicadores globales. Cabe destacar la meta 4.7 del ODS 4 "Educación de calidad" la cual recoge entre otros elementos que "De aquí a 2030, asegurar que todos los alumnos adquieran los conocimientos teóricos y prácticos necesarios para promover el desarrollo sostenible, entre otras cosas mediante la educación para el desarrollo sostenible y los estilos de vida sostenibles...".

El suelo es multifuncional y presta servicios vitales para las actividades humanas y la supervivencia de los ecosistemas, como son: la producción de alimentos y demás producción de biomasa; el almacenamiento, el filtrado y la transformación de nutrientes, agua, compuestos orgánicos, etc.; ser hábitat de una cantidad ingente de organismos, por tanto, reserva de la biodiversidad; ser plataforma de la mayor parte de las actividades humanas; ser elemento del paisaje; aportar materias primas; reserva de carbono; y almacenar el patrimonio arqueológico y geológico. Así, los suelos contribuyen a las necesidades humanas básicas como son la alimentación, el agua limpia y el aire limpio (Keesstra et al., 2016). La gestión de los suelos que lleva a su degradación o mejora tiene un impacto fundamental en otros campos como la protección de las aguas superficiales y subterráneas, la salud humana, el cambio climático, la protección de la naturaleza y la biodiversidad, y la seguridad alimentaria. 
El suelo es un recurso natural de interés común que se encuentra sometido a crecientes presiones medioambientales que pueden conllevar la pérdida de materia orgánica, la contaminación, la pérdida de la biodiversidad del suelo, el sellado, etc. El director general de la FAO, José Graziano da Silva, aseguró durante el $21^{\circ}$ Congreso Mundial de Ciencias del Suelo en Brasil, que mejorar la salud de los suelos del mundo es esencial para erradicar el hambre, y luchar frente al cambio climático y sus consecuencias.

La Carta Mundial de Naciones Unidas para la Naturaleza (1982) indica que se mantendrá o aumentará la productividad de los suelos con medidas de preservación de su fertilidad a largo plazo y de los procesos de descomposición orgánica y de prevención de la erosión y toda otra forma de deterioro.

De acuerdo con el Informe de los Objetivos de Desarrollo Sostenible 2019 (ONU 2019) "Las tendencias mundiales en cuanto a la cubierta terrestre indican una pérdida neta de clases de tierras naturales y seminaturales debido en gran medida a procesos inducidos por el hombre, como la desertificación, la deforestación, la gestión inadecuada de los suelos, la expansión de las tierras de cultivo y la urbanización". También se indica en dicho informe que "las pérdidas sustanciales en las reservas de carbono orgánico del suelo se produjeron generalmente durante las transiciones de la cubierta terrestre, tales como el cambio de un suelo arbolado a entornos construidos y tierras de cultivo".

El Informe de los Objetivos de Desarrollo Sostenible 2020 (ONU 2020) indica que "La degradación de los suelos afecta a miles de millones de personas, lleva a la extinción de especies e intensifica el cambio climático". Por tanto, es necesario revertir la tendencia actual de degradación de los suelos. También se indica en dicho informe que la superficie forestal del mundo sigue disminuyendo y que "Estos bosques perdidos significan la desaparición de los medios de vida de comunidades rurales, el aumento de las emisiones de carbono, la disminución de la biodiversidad y la degradación de suelos".

El informe de la FAO y GTIS (2016) "El estado mundial del recurso suelo" indica que la degradación del suelo es un problema mundial que está afectando a poblaciones en Norteamérica, Sudamérica, África, Asia y Europa. El informe identifica 10 amenazas principales a las funciones del suelo, incluida la pérdida de carbono del suelo. Es una prioridad mantener y aumentar las reservas de carbono del suelo.

Toda disciplina científica se enfrenta al desafío de actuar sobre los ODS, y esto es particularmente relevante para la ciencia del suelo, como una disciplina relacionada con la tierra con vínculos importantes con varios de los ODS (Keesstra et al., 2016). El suelo solo se menciona en 4 de las 169 metas, y no como una meta en sí (Lobry de Bruyn et al., 2017), sin embargo, muchos de los ODS no pueden alcanzarse sin suelos sanos y sin un uso sostenible del suelo.

De acuerdo con distintos autores, los ODS relacionados con el suelo son el ODS 2 (seguridad alimentaria), ODS 6 (calidad del agua), ODS 13 (mitigación del clima) y ODS 15 (preservación del ecosistema) (Bouma, 2019; Bonfante et al., 2020; Lal, 2021) (Figura 2). Lal (2018) clasificó los ODS relacionados con el suelo en aquellos que producen un impacto primario (ODS 2, ODS13 y ODS 15) y los que producen un impacto secundario (ODS 1, ODS 3, ODS 6 y ODS 7). Tóth et al. (2018) incluyen los ODS 11 y ODS 14 y también mencionan que los ODS 7 y 12 indirectamente se ven afectados por la salud de los suelos. También indica Lal (2018) que la salud de los suelos afecta a otros ODS a través de las interacciones con otras ecosferas, destacando el ODS 1, ODS 3 y ODS 16. La salud de los suelos afecta a la salud humana y al bienestar (ODS 3), como fuente de fármacos, a través de la exposición directa de los individuos a los suelos (Keesstra et al., 2016; Brevik et al., 2020; Steffan et al., 2020) y atenuando los contaminantes ambientales y patógenos (Porta et al., 2019). 


\begin{tabular}{|c|c|c|}
\hline ODS 2 & ODS 3 & ODS 6 \\
\hline $\begin{array}{l}\text { Mejorar la calidad del suelo para } \\
\text { acabar con el hambre y lograr la } \\
\text { seguridad alimentaria. }\end{array}$ & $\begin{array}{l}\text { Garantizar una vida sana y promover } \\
\text { el bienestar al evitar que los } \\
\text { productos químicos peligrosos y la } \\
\text { contaminación del aire y del agua } \\
\text { contaminen la el suelo. }\end{array}$ & $\begin{array}{l}\text { Garantizar la disponibilidad y la } \\
\text { gestión sostenible del agua y el } \\
\text { saneamiento para todos, } \\
\text { preservando la calidad del suelo } \\
\text { debido al papel que desempeña para } \\
\text { ayudar a proporcionar agua limpia } \\
\text { para beber y para la agricultura. }\end{array}$ \\
\hline ODS 11 & ODS 12 & ODS 13 \\
\hline $\begin{array}{l}\text { Hacer que las ciudades y los } \\
\text { asentamientos humanos sean } \\
\text { inclusivos y seguros, garantizando } \\
\text { que los suelos estén protegidos, ya } \\
\text { que salvaguardan nuestro } \\
\text { patrimonio cultural y natural. }\end{array}$ & $\begin{array}{l}\text { Garantizar patrones de consumo y } \\
\text { producción sostenibles, } \\
\text { asegurándose de que los productos } \\
\text { químicos y los desechos no lleguen } \\
\text { al suelo, lo que puede tener } \\
\text { impactos adversos en nuestra salud } \\
\text { y el medio ambiente. }\end{array}$ & $\begin{array}{l}\text { Tomar medidas urgentes para } \\
\text { combatir el cambio climático y sus } \\
\text { impactos. El suelo puede } \\
\text { desempeñar un papel positivo en la } \\
\text { reducción de los impactos del } \\
\text { cambio climático al secuestrar } \mathrm{CO}_{2} \text {. }\end{array}$ \\
\hline \multicolumn{3}{|l|}{ ODS 15} \\
\hline \multicolumn{3}{|c|}{$\begin{array}{l}\text { Proteger, restaurar y promover el uso sostenible de los ecosistemas terrestres, gestionar los } \\
\text { bosques de forma sostenible, combatir la desertificación, detener y revertir la degradación de } \\
\text { la tierra y detener la pérdida de biodiversidad. }\end{array}$} \\
\hline
\end{tabular}

Una de las amenazas de los suelos es la pérdida de materia orgánica. La materia orgánica del suelo se compone de materiales orgánicos, organismos vivos y humus; se encuentra en un proceso constante de acumulación y descomposición y su contenido resulta del balance entre los aportes y las pérdidas por descomposición. Con su descomposición se libera carbono a la atmósfera en forma de $\mathrm{CO}_{2}$, pero éste se recaptura mediante el proceso de fotosíntesis.

La materia orgánica es de gran importancia para que el suelo pueda realizar sus funciones y por tanto para la consecución de los ODS. Es fundamental que los suelos tengan un contenido adecuado de materia ya que da lugar a una buena estructura del suelo, las sustancias húmicas que la componen tienen un poder aglomerante, tiene una gran capacidad de retención de agua lo que facilita el asentamiento de la vegetación, la temperatura del suelo es mayor debido a que los colores oscuros absorben más radiaciones que los claros, adsorbe plaguicidas y otros contaminantes y evita que estos percolen hacia los acuíferos. La materia orgánica resulta un factor determinante de la resistencia a la erosión y de la fertilidad del suelo. La acumulación de materia orgánica en el suelo es un proceso lento (mucho más que el de pérdida de la misma). Es necesario mantener un tipo de gestión específico para conservar o incrementar el contenido de materia orgánica del suelo. La disminución de materia orgánica es especialmente preocupante en las regiones mediterráneas. 
Como indica (Keesstra et al., 2016) es necesario crear conciencia sobre la materia orgánica del suelo como un atributo clave de los suelos, ilustrando su importancia en el desarrollo de las funciones del suelo y los servicios de los ecosistemas.

\section{Objetivos}

El objetivo de este trabajo ha sido estructurar el aprendizaje por parte de los estudiantes de la relación entre las funciones del suelo y la materia orgánica con los ODS, así como el logro de resultados de aprendizaje en relación con las funciones de la materia orgánica. Para ello, en este trabajo, se describen las actividades que realizaron los estudiantes, así como los resultados de la prueba de evaluación en la que se incluyeron dos preguntas relacionadas con el suelo y los ODS.

\section{Desarrollo de la innovación}

La innovación se llevó a cabo con el alumnado matriculado en la asignatura de Medio abiótico de segundo curso del Grado en Ciencias Ambientales de la Universitat Politècnica de València en el curso académico 2020-2021. El número total de estudiantes matriculados era 38. En la asignatura se imparten tres materias: Edafología, Meteorología e Hidrología. La innovación docente se realizó en la parte de Edafología.

Los 38 estudiantes hicieron un cuestionario a principio de curso sobre los ODS. En el aula, los estudiantes elaboraron un esquema con la composición y propiedades de la materia orgánica y en el incluyeron la relación entre la materia orgánica y los ODS, y como actividad fuera del aula tenían que leer un texto para comentar y relacionar la materia orgánica y su manejo con los ODS. Dicho texto fue un extracto del epígrafe "Pérdida de materia orgánica" del documento "Hacia una estrategia temática para la protección del suelo" (Comisión Europea, 2002). Esta actividad puntuable fue entregada por 18 estudiantes. Esta actividad tenía una doble finalidad, que los estudiantes fijaran conocimientos sobre la materia orgánica y que vieran la importancia de la materia orgánica del suelo para la consecución de algunos de los ODS. Por último, en el examen final, dos de las cuestiones a desarrollar por los estudiantes fueron: 1) comentar las funciones de los suelos y su asociación con los ODS 2, ODS 3, ODS 6, ODS 13 y ODS 15, y 2) comentar la importancia de la materia orgánica para la consecución de los ODS 2, ODS 3, ODS 6, ODS 13 y ODS 15.

\section{Resultados}

Los estudiantes de esta asignatura ya tenían conocimientos sobre los ODS ya que éstos se comentan de forma introductoria en las asignaturas de primer curso "Medio Ambiente y Sociedad" y "Economía y Política Ambiental". Los estudiantes realizaron un test (Leiva-Brondo et al., 2020) sobre los ODS al principio de la asignatura. En este test participaron los 38 estudiantes matriculados y mostraron un alto conocimiento de los ODS (datos no presentados en el presente estudio).

La actividad en la que los estudiantes tenían que relacionar la materia orgánica con los ODS y que conllevaba una lectura inicial de un texto sobre la pérdida de materia orgánica fue realizada por el 48,6\% de los estudiantes. La importancia de la lectura y comentario del texto propuesto radica en que proporciona información relacionada con algunos de los resultados de aprendizaje de la Edafología a lograr por los estudiantes, entre ellos: identificar los distintos componentes de la materia orgánica, argumentar la necesidad de incorporar materia orgánica a los suelos, argumentar la necesidad de que se utilicen técnicas adecuadas de gestión de explotaciones que conlleven un aumento de la materia orgánica o eviten su pérdida, 
explicar el papel de los suelos en el ciclo de carbono y el papel clave de los suelos en el cambio climático teniendo en cuenta la captura de carbono en los suelos y su liberación de $\mathrm{CO}_{2}$. Estos resultados de aprendizaje se pueden evaluar a través de las relaciones razonadas que establecen los estudiantes con los ODS y también puede ser evaluado su logro con la pregunta de examen sobre la importancia de la materia orgánica para la consecución de algunos ODS.

La nota media de la actividad fue de 7,4 $\pm 2,5$ (media \pm desviación estándar). El 33,3\% de los estudiantes que hicieron la actividad sacaron la máxima nota. El 88,9\% de los estudiantes relacionaron la materia orgánica con el ODS 2, un 83,3\% con el ODS 6 y con el ODS 13 (Figura 3). Cabe resaltar que a pesar de ser la materia orgánica uno de los componentes principales de los suelos, un $22 \%$ de los estudiantes no la relacionaron con la vida de los ecosistemas terrestres (ODS 15).

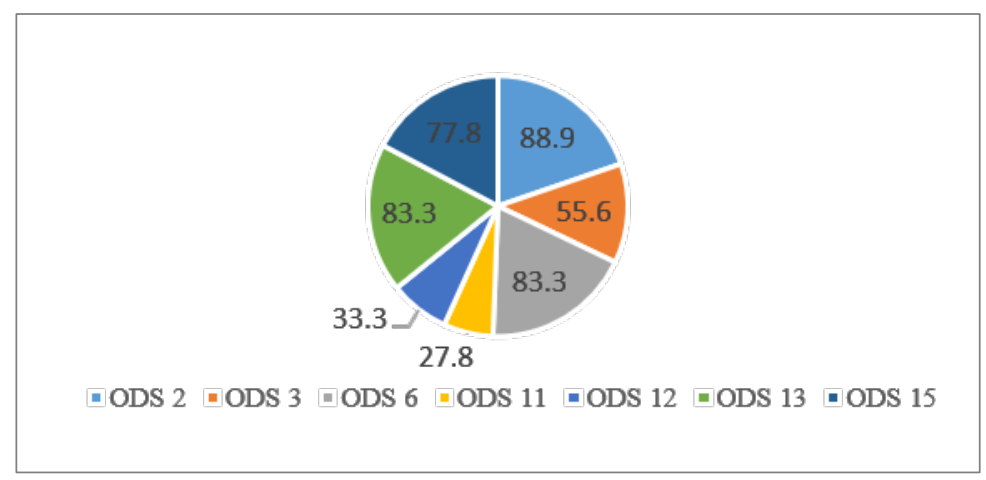

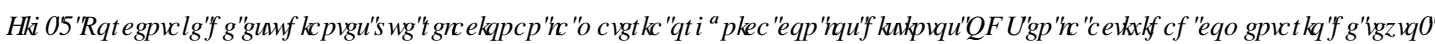

En el examen de Edafología se pidió al alumnado que comentara las funciones de los suelos y su asociación con los ODS 2, ODS 3, ODS 6, ODS 13 y ODS 15. La nota media fue de 8,0 $\pm 2,6$ (media \pm desviación estándar). Tan solo dos estudiantes hicieron una breve introducción nombrando la Agenda 2030: "Todas las acciones que se lleven a cabo en la Agenda 2030 deben tener la perspectiva del cuidado y protección del suelo para que podamos seguir habitando el planeta" (Al 26), "Los ODS están compuestos por más de 200 [son 169] metas que se quieren alcanzar antes del año 2030. Los países firmantes se comprometen a hacer todo lo posible por cumplir los ODS... Lo que se pretende es tener un futuro donde las personas puedan tener una vida digna" (Al 9). Uno de los estudiantes hizo mención a los suelos del marjal de Gandía al comentar la materia orgánica y el ODS 13, esto es interesante ya que la asociación de un ODS con un caso práctico real es necesario para alcanzar la concienciación social y medioambiental que subyace en los ODS.

La Tabla 1 recoge una clasificación de las funciones de los suelos necesarias para la consecución de los ODS extraída de las respuestas de los estudiantes, así como el número de estudiantes que comentan cada una de dichas funciones. La mayoría de los estudiantes relacionaron la función producción de alimentos con el ODS 2 (73\%), seguido de la función de ser hábitat de organismos y reserva genética con el ODS 15 $(64,9 \%)$ y un $54 \%$ relaciona la función secuestro de carbono con el ODS 13. Como puede observarse en la Tabla 1 es el ODS 3 el que los estudiantes han relacionado con un mayor número de funciones del suelo. La salud y bienestar están relacionados con el consumo de alimentos en cantidad y calidad sufícientes, con el acceso a agua limpia, con la obtención de medicamentos a partir de los organismos de los suelos, con el disfrute del suelo como elemento del paisaje y del patrimonio cultural y con la mitigación del cambio climático.

La relación entre las funciones del suelo y la disponibilidad de agua (ODS 6) no se obvia (Tabla 2). En el caso del ODS 6, el 64,9\% de los estudiantes indicaron que el suelo es un filtro natural del agua, pero no especificaron a que se debe (Tabla 2). Los suelos sirven de filtro natural de las aguas subterráneas, que son 
la principal reserva de agua potable. Los suelos son filtros de las aguas debido a : 1) las partículas del suelo que actúan como un filtro y limpian el agua a medida que se infiltra (proceso físico), 2) la adsorción de contaminantes debido a las cargas negativas y positivas que tiene algunas partículas del suelo, gracias a la adsorción los contaminantes quedan retenidos y se puede evitar que lleguen a nuestras aguas subterráneas (proceso químico) (ocho estudiantes mencionaron este proceso), 3) los microorganismos del suelo pueden transformar y/o descomponer productos químicos orgánicos peligrosos (proceso biológico). Por tanto, los suelos mantienen y mejoran la calidad de las aguas que lo atraviesan: al controlar el filtrado, los intercambios iónicos, el almacenamiento y la posible transferencia a otro compartimento ambiental (filtro ambiental). Solo el 21,6\% de los estudiantes relacionaron el agua limpia con la adsorción de contaminantes por parte del suelo y un $13,5 \%$ con la actividad de degradación de contaminantes por los microorganismos del suelo. Solo dos estudiantes relacionaron la función almacén de agua con el ODS 6.

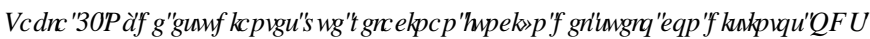

\begin{tabular}{|c|c|c|c|c|}
\hline Funciones de los suelos & $\begin{array}{c}\text { ODS } 2 \\
\text { Hambre cero }\end{array}$ & $\begin{array}{l}\text { ODS } 3 \text { Salud y } \\
\text { bienestar }\end{array}$ & $\begin{array}{l}\text { ODS } 13 \text { Acción } \\
\text { por el clima }\end{array}$ & $\begin{array}{c}\text { ODS 15 Vida } \\
\text { ecosistemas terrestres }\end{array}$ \\
\hline $\begin{array}{l}\text { Producción de alimentos } \mathrm{y} \\
\text { biomasa }\end{array}$ & 27 & 5 & & \\
\hline Filtro natural del agua & & 10 & & \\
\hline $\begin{array}{l}\text { Hábitat de organismos y reserva } \\
\text { genética }\end{array}$ & & & & 24 \\
\hline $\begin{array}{l}\text { Entorno físico y cultural para el } \\
\text { ser humano }\end{array}$ & & 8 & & \\
\hline $\begin{array}{l}\text { Atenuar los contaminantes } \\
\text { ambientales y patógenos }\end{array}$ & & 10 & & \\
\hline Obtención de medicamentos & & 2 & & \\
\hline Proporciona materias primas & 2 & 1 & & \\
\hline Mitigación cambio climático & & 2 & & \\
\hline - Fija gases efecto invernadero & & & 13 & \\
\hline - Secuestro de carbono & & & 20 & \\
\hline - Regula el microclima & & & 14 & \\
\hline No relacionan con ODS & 8 & 11 & 5 & 3 \\
\hline
\end{tabular}

El número de estudiantes que realizaron el examen fue 37.

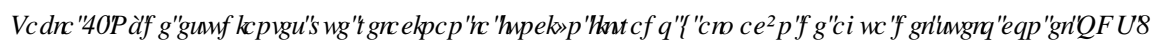

\begin{tabular}{clc}
\hline Filtrado natural del agua & 24 \\
$-\quad$ Partículas del suelo (físico) & 0 \\
$-\quad \begin{array}{l}\text { Adsorción de contaminantes } \\
\text { (químico) }\end{array}$ & 8 \\
$-\quad \begin{array}{l}\text { Acción } \\
\text { descomposición) } \begin{array}{l}\text { de de lonsformación, } \\
\text { microorganismos del suelo sobre los } \\
\text { contaminantes (biológico) }\end{array}\end{array}$ \\
\hline Almacén de agua & 5 \\
\hline No relacionan con ODS 6 & 2 \\
\hline
\end{tabular}

El número de estudiantes que realizaron el examen fue 37.

(c)) BY-NC-ND 2021, Universitat Politècnica de València

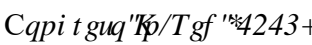


Otra cuestión planteada en el examen de Edafología fue: ¿por qué la materia orgánica es importante para la consecución de los ODS 2, ODS 3, ODS 6, ODS 13 y ODS 15? La nota media fue de 5,8 $\pm 2,8$ (media \pm desviación estándar) e indica un menor aprendizaje. Destacar que la nota media obtenida del promedio de los estudiantes que hicieron la actividad relativa a comentar y relacionar la materia orgánica con los ODS y que incluía la lectura de un texto sobre la perdida de materia orgánica fue 1,9 puntos superior a la de los alumnos que no la realizaron.

Los suelos sanos son clave para la seguridad alimentaria. El 95\% de los alimentos vienen del suelo. El 84\% de los estudiantes indicaron que, al aumentar la materia orgánica en el suelo, los suelos son más fértiles y se pueden obtener más alimentos (Figura 4). Un $10.8 \%$ comentaron que la materia orgánica mejora las propiedades físicas del suelo (p. ej., estructura, porosidad) y que esto afecta a la calidad del suelo y por tanto a la obtención de alimentos. Un 8.1\% comentaron que en los suelos con materia orgánica disminuye la erosión y por tanto la pérdida de suelo y una posible contaminación de las aguas por eutrofización. Solo un estudiante indicó que se puede añadir materia orgánica a los suelos degradados para recuperarlos y poder utilizarlos para cultivar.

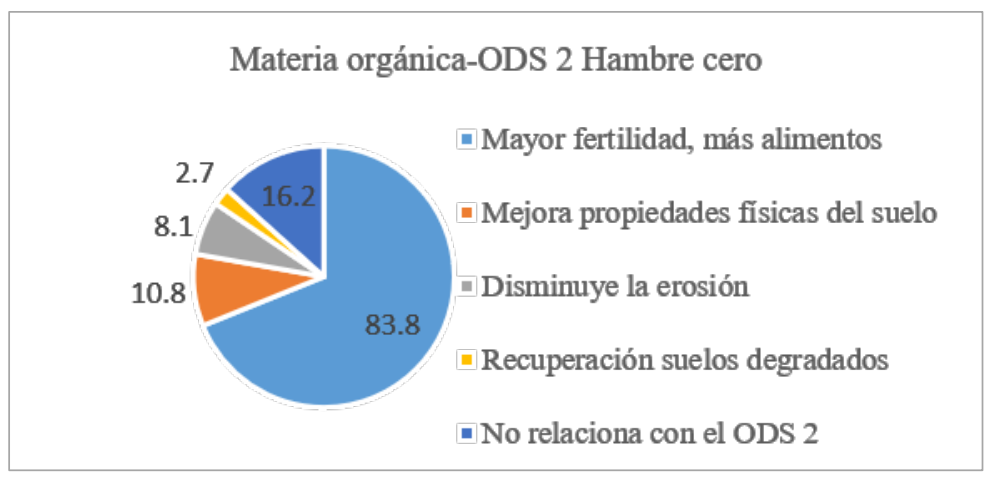

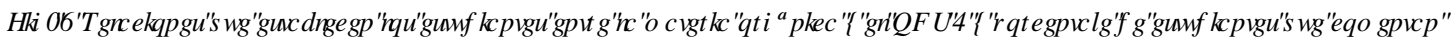 FDODIXQDICHCOVLUDFIRQHW}

Un 37.8\% de los estudiantes no relacionaron la materia orgánica con el ODS 3 (Figura 5), sin embargo, un $45.9 \%$ indicaron que al aumentar el contenido en materia orgánica aumenta la retención de contaminantes en el suelo debido a la capacidad de adsorción de la materia orgánica del suelo, impidiendo que estos contaminantes lleguen al agua subterránea y pueda producir enfermedades y un $16.2 \%$ de los estudiantes comentaron que afecta a la salud relacionándolo con la función del suelo de producir alimentos.

\section{Materia orgánica-ODS 3 Salud y bienestar}

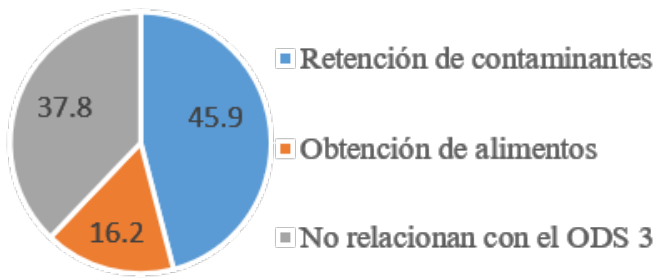

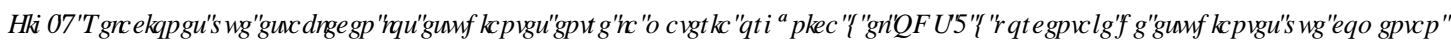
FDODXQDGHOWUHDFIRQHW

Un 51.4\% (Figura 6) de los estudiantes comentaron la capacidad de los suelos de retener contaminantes y evitar así su lixiviación a aguas subterráneas, un 27\% indicaron que la materia orgánica del suelo ayuda en 
el papel de suelo de ser filtro natural de las aguas, pero no explican el motivo. Un $13.5 \%$ de los estudiantes indicaron que al tener un suelo más materia orgánica, el suelo retiene más agua, lo cual es beneficiosos para el desarrollo de la vegetación y el 10.8\% nombraron el papel de la materia orgánica en la disminución de la erosión de los suelos y su relación con la eutrofización.

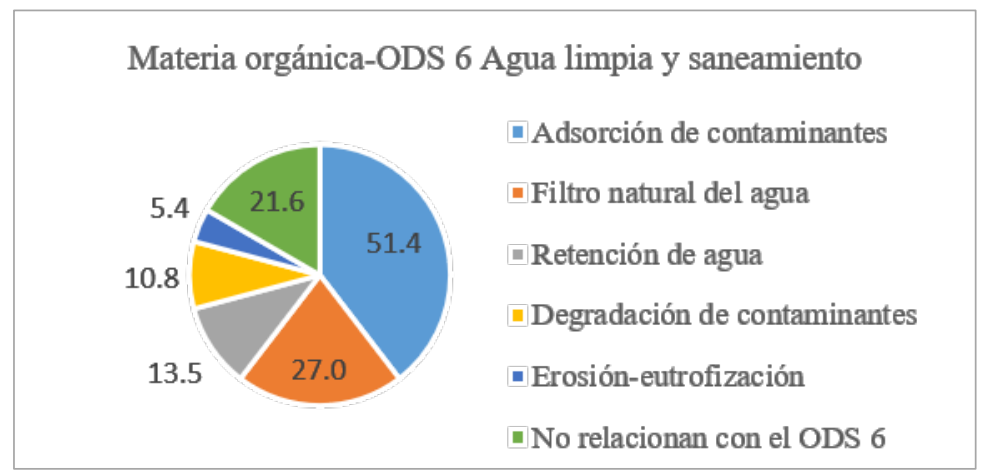

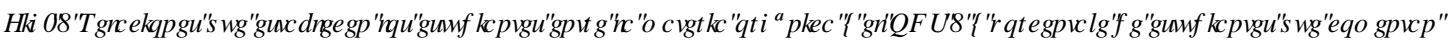 FDODXQDICHHMVUUHDFRQHW}

Los suelos tienen un papel clave en la mitigación del cambio climático. El 37.8\% de los estudiantes asocian este papel de los suelos y más en concreto de la materia orgánica con el secuestro de carbono y solo un $5.4 \%$ lo asocia a que el suelo es un sumidero de gases de efecto invernadero (Figura 7). Uno de los resultados de aprendizaje era explicar el papel de los suelos en el cambio climático teniendo en cuenta que el suelo puede secuestrar carbono. La mayoría de estudiantes comentan que el suelo secuestra carbono, pero no lo razonan. Un alto porcentaje (35.1\%) no contestó a la relación de la materia orgánica con el ODS 13 o lo hizo erróneamente.

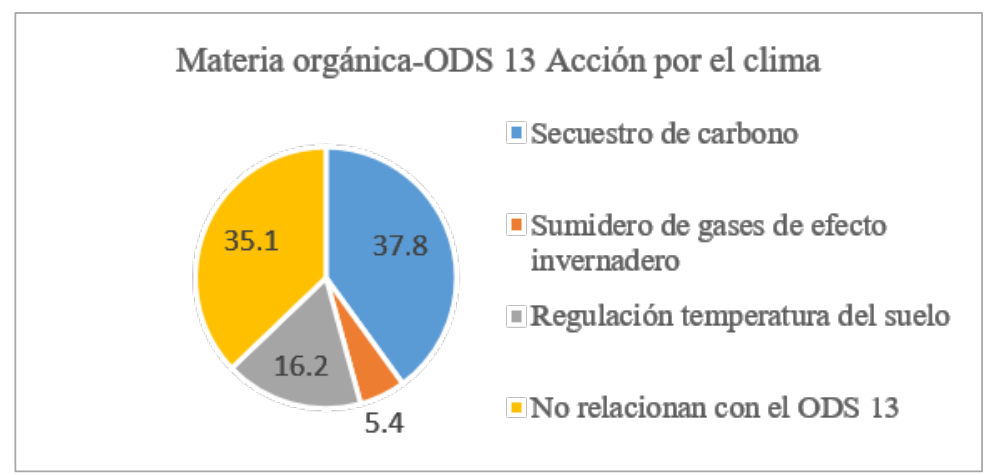

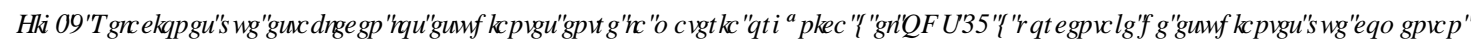 FDODXQDIGHHWVUHDFIRQHM}

Por último, destacar que un 37.8\% (Figura 8) indicaron que cuanto mayor es la materia orgánica mayor es el número de organismos que hay en suelo y debido a que hay más nutrientes en el suelo procedentes de la descomposición de la materia orgánica [humificación y mineralización] hay mayor vegetación. La mayoría de estudiantes mencionan a los microorganismos entre esos organismos. Hay que tener en cuenta que los microorganismos del suelo o biomasa microbiana forma parte de la materia orgánica del suelo. 


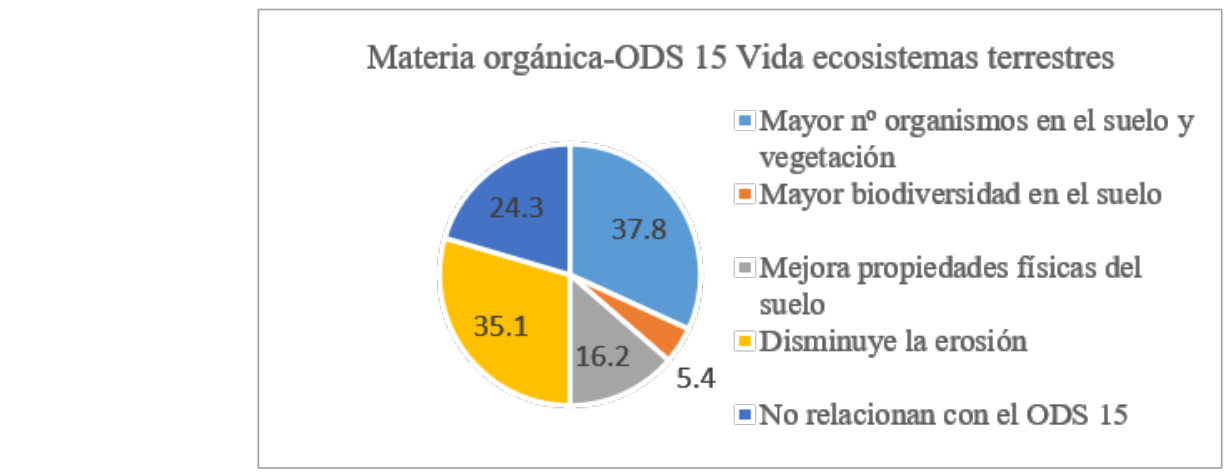

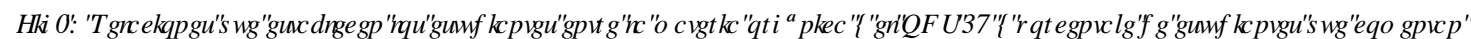 FDODXQDGHHWDULDDFRQHM}

Un $35.1 \%$ de los estudiantes comentaron que cuanto mayor es el contenido en materia orgánica disminuye la erosión del suelo y por tanto la posible eutrofización de las aguas de ríos, lagos, etc. Un $16.2 \%$ asocia la materia orgánica a una mejora de las propiedades físicas, como la estructura y la porosidad, en relación a la vida vegetal.

A través de la relación de la materia orgánica con los ODS algunos estudiantes mostraron haber adquirido los resultados de aprendizaje definidos para la materia orgánica en la asignatura de Medio abiótico. Esto queda reflejado en los razonamientos de los estudiantes para asociar los ODS con la materia orgánica y sus funciones y que quedan recogidos de manera simplificada en las Figuras 4 a 8.

En los últimos años ha habido una proliferación de artículos de investigación que relacionan el suelo y los ODS sin embargo es necesario también que se divulguen artículos de investigación educativa con buenas prácticas que desarrollen el papel de los suelos en la consecución de los ODS.

\section{Conclusiones}

En este trabajo se ha expuesto la inclusión de los ODS en la materia de Edafología impartida a estudiantes de segundo curso del grado en Ciencias Ambientales, a través de una actividad y su incorporación a la evaluación. De los resultados obtenidos se observa que los estudiantes establecen un grado alto de relación de las funciones de los suelos con los ODS. Sin embargo, esta relación es menor en el caso de la materia orgánica y los ODS, lo que confirma que se debe de trabajar más en el aula. La actividad realizada sobre la materia orgánica y los ODS tuvo una repercusión positiva en el aprendizaje sobre el papel de la materia orgánica en los suelos. El promedio de la nota de los estudiantes que hicieron dicha actividad fue dos puntos superiores a la de los alumnos que no la realizaron. Destacar que es necesario para incorporar objetivos de sostenibilidad en el proceso de enseñanza-aprendizaje poner ejemplos prácticos y realistas.

\section{Agradecimientos}

La publicación de este trabajo ha sido parcialmente financiada por el proyecto de innovación educativa (PIME 20-21/224) concedido por el Vicerrectorado de Estudios, Calidad y Acreditación de la Universitat Politècncia de València (UPV). 


\section{Referencias}

BONFANTE, A., BASILE, A., BOUMA, J. (2020). "Targeting the soil quality and soil health concepts when aiming for the United Nations Sustainable Development Goals and the EU Green Deal" en 6RIQ6 (2), 453-466.

BOUMA, J. (2019). "How to communicate soil expertise more effectively in the information age when aiming at the UN Sustainable Development Goals" en 6RIOB HHDQGO DQDJHPHQW35 (1), 32-38.

BREVIK, E. C., SLAUGHTER, L., SINGH, B. R., STEFFAN, J. J., COLLIER, D., BARNHART, P., PEREIRA, P. (2020). "Soil and human health: current status and future needs" en \$IU] 6RIQ DQG : DUHU 5HMDF, पI, 1178622120934441 .

COMISIÓN EUROPEA. 2002. Comunicación de la Comisión al Consejo, el Parlamento Europeo, el Comité Económico y social y el Comité de las Regiones. Hacia una estrategia temática para la protección del suelo. Doc COM (2002) 179 .

FAO y GTIS. Organización de las Naciones Unidas para la Alimentación y la Agricultura y Grupo Técnico Intergubernamental del Sue. (2016). Estado Mundial del Recurso Suelo. Resumen Técnico. Roma. $<$ http://www.fao.org/3/I5126S/i5126s.pdf> [Consulta: 10 de febrero de 2021]

KEESSTRA, S. D., BOUMA, J., WALLINGA, J., TITTONELL, P., SMITH, P., CERDÀ, A., MONTANARELLA, L., QUINTON, J. N., PACHEPSKY, Y., VAN DER PUTTEN, W. H., BARDGETT, R. D., MOOLENAAR, S., MOL, G., JANSEN, B., FRESCO, L. O. (2016). "The significance of soils and soil science towards realization of the United Nations Sustainable Development Goals” en 6RIQ2 (2), 111-128.

LAL, R. (2018). "Sustainable Development Goals and the Intenational Union of Soil Sciences" en Lal, R., Horn, R., Kosaki, T. 6RIODQC6 6XWQDEOH' HHRSP HQW* RDO. Catena-Scheizerbart: Stuttgart, Alemania. p. 189-196.

LAL, R. (2021). "Soil Strength and Carbon Sequestration" en Hunt, A., Egli, M., Faybishenko, B. +|GRJHRRJ\ \&KH IFDO. HDKHUD

LEIVA-BRONDO, M., ATARÉS, A., PÉREZ-DE-CASTRO, A., LULL, C. (2020). Preliminary assessment of Sustainable Development Goals by freshmen students of a life science degree. INTED2021 Proceedings 15th International Technology, Education and Development Conference. Marzo 8-9, 2021.

LOBRY DE BRUYN, L., JENKINS, A., SAMSON-LIEBIG, S. (2017). "Lessons learnt: Sharing soil knowledge to improve land management and sustainable soil use" en 6RIO6FHQFH6RFIHMRILS P HIFD-RXLQDQ81(3), 427-438.

ORGANIZACION DE LAS NACIONES UNIDAS (ONU). (2019). Informe de los Objetivos de Desarrollo Sostenible 2019. <https://unstats.un.org/sdgs/report/2019/The-Sustainable-Development-Goals-Report-2019_Spanish.pdf $>$ [Consulta: 8 de febrero de 2021]

ORGANIZACION DE LAS NACIONES UNIDAS (ONU). (2020). Informe de los Objetivos de Desarrollo Sostenible 2020. <https://unstats.un.org/sdgs/report/2020/The-Sustainable-Development-Goals-Report-2020_Spanish.pdf $>$ [Consulta: 8 de febrero de 2021]

PORTA, J., POCH, R. M., LÓPEZ-ACEVEDO, M. (2019) Edafología: uso y protección de suelos. $4^{\mathrm{a}}$ Edición. Ediciones Mundi-Prensa, Madrid 624p.

STEFFAN, J. J., DERBY, J. A., BREVIK, E. C. (2020). Soil pathogens that may potentially cause pandemics, including SARS coronaviruses" en \&XUHQID SIQRQIQR QVILRQP HQWDOFFHQFHI I+ HDOK, 17, 35-40.

TÓTH, G., HERMANN, T., DA SILVA, M. R., MONTANARELLA, L. (2018). "Monitoring soil for sustainable development and land degradation neutrality” en ( QVILRQP HQWDOP RQUTRUQJDQCFDMHMP HQW190[(2), 1-4. 